\title{
Effect of Early Rehabilitation Program on Mechanically Ventilated Patient's Outcomes
}

\author{
Safaa Mohamed Adam, Khaled Mohamed Abd El-Hameed \& Mona Aly Mohammed. \\ Demonstrator in Critical Care Nursing and Emergency Department, Faculty of Nursing, Sohag University Egypt. \\ Professor of Anesthesia and Intensive Care, Faculty of Medicine, Sohag University Egypt. \\ Assistant Professor in Critical Care Nursing, Faculty of Nursing, Assuit University Egypt.
}

\begin{abstract}
Background: Rehabilitation is a cornerstone in management of mechanically ventilated patients in ICU concerned reducing impairments, preventing complications and reduce weaning time. Therefore, the aim was to identify the effect of early rehabilitation on mechanically ventilated patient's outcomes. Quasi experimental research design was used to conduct this research. This study was carried out at general intensive care unit at Sohag University Hospitals. The sample of this study was consisted of 60 patients, divided into study and control group equally. Tools used in this study; Tool I: Modified patient assessment sheet, Tool II: patients complications assessment sheet, Methods: Each patient of the study group subjects were exposed in addition to routine hospital care to two sessions/day of rehabilitation program until disconnection from mechanical ventilator. However control group subjects were exposed to routine hospital care only. The main results:- Finding of the present study revealed a significant statistical differences $(\mathrm{P}<0.05)$ found between both groups in relation to improvement on oxygenation, length of stay on mechanical ventilation, length of ICU stay, respiratory infection, and weaning in study group compared to control group. Conclusion: Applying early rehabilitation for mechanically ventilated patient help to reduce complications and improve the outcomes. Recommendation: Further researches are recommended to include information about the follow-up of these patients out of ICU.
\end{abstract}

Keywords: Rehabilitation, Early Mobilization \& Weaning\& Patient's Outcomes.

\section{Introduction}

Therapeutic processes in ICU often require the application of mechanical ventilation, which necessitates intubation or tracheostomy. The activities performed upon the patient involve comprehensive therapy, in which rehabilitation has been considered to be an integral part of treatment for mechanically ventilated patients. (Mączka et al., 2011) Rehabilitation in the ICU has been defined in a position paper by The European Respiratory Society as a process to achieve optimal daily functioning and health-related quality of life of individual patients as measured by clinically and/or physiologically relevant outcome measures. (Denehy \& Berney., 2006 )

"Early" refers to rehabilitation interventions that commence immediately after stabilization of physiologic derangements, often while patients remain on mechanical ventilation and vasopressor infusions. (Parker \& Needham, 2013) continued throughout the ICU stay. Such activities may start within 1 or 2 days of initiation of mechanical ventilation. (Mendez-Tellez et al., 2012)

Rehabilitation goal does not only comprise the patient's individual perspective, but also his environment, family, or any other persons involved. (Müller et al., 2011), Rehabilitation is focused not on permanently removing the patient from the ventilator, but on improving the patient's capacity for independent activity of daily living and mobility. (Make et al., 1984) may reduce the risk of difficult weaning, limited mobility and ventilator dependency. (Ambrosino et al., 2012) With the help of education, the rehabilitation also aims to relieve fears and anxiety associated with program and lung condition, thereby ensuring long-term commitment to exercise. (Sharma and Singh, 2011)

Rehabilitation program included mobilization, positioning, continuous rotational therapy, respiratory muscle training, manual hyperinflation, percussion and vibrations, suction and, incentive spirometry (Ambrosino et al., 2012) education, breathing exercise, and psychosocial support (Dong et al., 2014).

Postural drainage refers to placing the body in a position that allows gravity to assist drainage of mucus from the lung periphery to the segmental bronchus and upper airway. (Ciesla, 2014)

Chest percussion is also referred to as cupping, clapping, and tapotement. The purpose of percussion is to intermittently apply kinetic energy to the chest wall and lung. This is accomplished by rhythmically striking the thorax with cupped hand or mechanical device directly over the lung segment(s) being drained (Irimia, 2012). Vibration is a fine, shaking pressure applied to the chest wall during the exhalation only. This technique increases the velocity 
and turbulence of exhaled air, facilitating removal of secretions. Vibration increase exhalation of trapped air, shakes mucus loose, and induces a cough. (Perry et al., 2015)

Manual hyperinflation ( $\mathrm{MH})$ is commonly applied in patients under MV. It may stimulate cough and move the airway secretions toward the larger airways, from where they can be easily suctioned. Manual hyperinflation can prevent airway plugging and pulmonary collapse, and improve oxygenation and lung compliance. (Ambrosino \& Makhabah., 2014) Patient with an endotracheal tube (ETT) or tracheostomy tube require suction to remove pulmonary secretions that can lead to atelectasis or airway obstruction and impaired gas exchange. Suction should be performed as clinically indicated, with assessment of visible or audible secretions, rising inspiratory pressure, decreasing tidal volume or increase work of breathing. A saw-tooth pattern on the flow-volume waveform may indicate the need for suction. (Elliot et al., 2012)

Education represents a very important component of the rehabilitation process.(Carlin, 2009) .The implications of mechanical ventilation are frequently misunderstood and inappropriately feared, educational sessions on the nature and outcomes of life-supportive care are considered valuable and are well received by the majority of patients with chronic disease. Many patients otherwise carry a misperception that once they are intubated, they will never again have the ability to breath on their own. Educational curricula can provide information on the indicators for use and the mechanics of assisted ventilation. (Heffner \& Byock, 2002)

Psychological and social support: chronic illness, the need for mechanical ventilation, progressive disability, and increased dependence create conflicts both for patients and for their families. Such conflicts must be considered in patient management, and specialists in all the disciplines of the rehabilitation team help in this area. The active participation of patients and their families in establishing a care plan is encouraged. (Avendaño \& Güell, 2003)

Positioning the intensive care ventilated patient can improve patient comfort and also address the physiological aims of optimizing oxygen transport (through the effects of improving ventilation/ perfusion mismatching), reducing the work of breathing and reducing myocardial workload (Clini \& Ambrosino, 2005).

Mobilization in the ICU is defined as a progressive continuum in which critically ill patients are assessed for readiness to tolerate activity and/or movement, based on the patient's specific diagnosis and care needs, and are safely mobilized in order to reduce complications from inactivity to improve patient outcomes (Vollman., 2013).

Critical care nurses play an important role in rehabilitation; she is acting as the primary link for the patient and family. They also take responsibility for providing specialist information and advice, coordinating referrals and liaising with other professionals as necessary. (Rooney., 2013), using the nursing process, the nurse develops a plan of care designed to facilitate rehabilitation, restore and maintain optimum health, and prevent complications. Provides frequent passive range-of-motion exercises if the patient is unable to perform active exercises. (Hinkle and Cheever, 2014)

\section{Operational Definitions}

\section{Rehabilitation}

Rehabilitation is the process of restoring health or normal life by training and therapy after illness through decrease bed rest complications and patients' ventilator dependency; improve residual function prevent the need for new hospitalizations; and to improve health status

\section{Patient's outcomes}

Improve weaning outcome, length of intensive care unit stay, prevent or decrease ventilator complication and, decrease ventilator period.

\section{Significance of the study}

Statistics of intensive care unit at Sohag university hospital in the years of 2013 \& 2014 revealed that the number of patients admitted to intensive care unit were approximately 350 patients. (75\% of them were connected to mechanical ventilation). (Hospital records of Sohag University 2013-2014). Clinical observation of researcher revealed that most of those patients were suffering from a lot of respiratory and other problems which might endanger their life, increase hospital stay and the period of connection to the mechanical ventilator. Despite these complications can be minimized or prevented.

Therefore :- The objective of this study To determine effect of early rehabilitation on mechanically ventilated patient's outcome as indicated by weaning, length of intensive care unit stay, ventilator complications, ventilator period. This study could be beneficial in many ways; first it will help to apply advanced cost effective therapeutic tools to decrease bed rest complications and patients' ventilator dependency; second to improve health status and quality of life; third and last it helps to prevent weaning failure. 


\section{Aim of the study}

The aim of this study: was to determine effect of early rehabilitation on mechanically ventilated patient's outcomes

Specific objectives

- Reduce length of intensive care unit stay, ventilator complications, and ventilator period.

- Improve the subsequent health-related quality of life.

- Achieve successful weaning.

- improve residual function

Hypothesis

Hypotheses: To fulfill the aim of the study the following research hypothesis were formulated:-

Hypothesis (1) the post mean homodynamic parameters of study group will be better than those of the control group.

Hypothesis (2) the excepted mean arterial blood gases values of the study group will be better than those of the control group.

Hypothesis (3) the frequency of complications will be lesser among study group subjects compared to those of control group

Hypothesis (4) the frequency of length of stay on mechanical ventilation and length of stay on ICU will be lesser among study group subjects compared to those of control group

\section{Subjects and methods}

Research design: quasi experimental research design was used to conduct this study.

Setting: The study was carried out in intensive care units at Sohag university hospital.

Subjects: A purposive sample of 60 critically ill patients in critical care units. All patients were admitted to intensive care unit on mechanical ventilation. They were divided into equal 30 patients for both study and control group.

Inclusion Criteria: newly admitted to intensive care unit on mechanical ventilation without complications, age $>18$ years old.

Exclusion criteria: Excluded from this study patients had history of recurrent admission to ICU connected to mechanical ventilation, or admitted with septic shock, neurological injury orthopedic injury with a contraindication to mobilize and long term mechanical ventilation

Tools

First Tool: Modified Patient assessment sheet: -

This tool was developed by the researcher based on reviewing of the relevant literature and used to assess the studied patients regard the socio-demographic and medical data as base line data, it includes two main parts as flowing:-
Part one: patient characteristic

This part includes socio- demographic and clinical data of the patient as (code, age, sex, level of education, History of current disease, past medical diseases, date of admission and medical diagnosis, number of days on mechanical ventilator, length of ICU stay, weaning duration ,numbers of spontaneous breathing trails)

Part two: this part consists of following categories: - Assessment of hemodynamic parameters:- It includes temperature, heart rate, blood pressure \& mean arterial pressure, central venous pressure reading.

- Assessment of the ventilator parameters: It includes, tidal volume (vt), respiratory rate $(\mathrm{F})$, fraction of inspired oxygen $\left(\mathrm{Fio}_{2}\right)$, positive end expiratory pressure (PEEP), rapid shallow breathing index

- Laboratory investigation:- It include blood gases measurements, blood picture, liver function test, renal function test, and serum electrolytes.

- Respiratory system assessment:-In terms of the following (breathe sounds, secretions, colour and viscosity)

- Neurological assessment:- By Richmond agitation sedation scale (Sessler et al., 2002) that measures the sedation or agitation of ICU patients. It is a 10point scale, with four levels of anxiety or agitation, one level denoting a calm and alert state, and 5 levels of

- Assess level of exertion by Borg Rate of Perceived Exertion The Borg Rate of Perceived Exertion is designed to describe perceptions of physical exertion during a wide range of exercise modes. The scale consists of numbered categories, $0-10$, and verbal cues, from "Nothing at all" to "Maximal exertion. (Borg, 1998)

Second Tool: patients complications assessment sheet

This tool was developed by the researcher based on review of related literature

Part one: that covered assessment clinical adverse event that could have been associated with the exercise (new cardiac arrhythmias, hemodynamic instability or self extubation).

Part 2: this part covered short term complication from mechanical ventilation such as:-

Deep venous thrombosis (DVT): (calf pain, calf tenderness, warm calf, swelling of extremities, redness, concerned with dobbler).

Weaning failure: a) failure of spontaneous breathing trail (SBT) or b) the need for reintubation within 48 hrs. Following extubation. Failure of SBT is defined by such as tachypnea, tachycardia, hypertension, hypotension, hypoxemia or acidosis, arrhythmia, 
distress, and diaphoresis, c) Death within $48 \mathrm{~h}$ following estuation

Ventilator associated- lung injury by using lung injury scale developed by (Maskara 2000) that designed to assess patient for the presence and extent of a pulmonary damage. It can be used both at the onset of a lung disorder and during the course of the illness to monitor changing lung involvement. Parameters used are 1-chest X-ray evaluated for alveolar consolidation (2) ratio of the partial pressure of oxygen in arterial blood to the inspiratory fraction of oxygen (3) PEEP level if ventilated(4) respiratory compliance .The score was calculated based on the results of logistic regression analysis. Score $=$ sum values parameters maximum summation of parameters $=16$, minimum summation of parameters $=0$, score 0 : no lung injury, score $0.1-2.5$ : mild-tomoderate lung injury, score $>2.5$ : severe lung injury (ARDS).

Ventilator associated pneumonia (The standard diagnostic criteria in VAP include at least two of the following three findings: fever, leukocytosis or Leucopenia $(<4,000 / \mathrm{mm} 3)$ and purulent tracheal secretions, usually with abnormal findings from chest radiographic studies)

\section{Operational design}

The study was carried out on two phases

Preparatory phase

An official Permission was granted by the researcher from the head of intensive care unit at Sohag university hospitals after explanation the aim and nature of the study.

\section{Content validity}

The tools were tested for content related validity by specialists in the field of critical care medicine and critical care nursing from Sohag \& Assuit University, and the necessary modifications were done.

\section{Pilot study}

A pilot study was carried out on 6 patients to test the clarity and applicability of the tools and time needed to collect the data. The tools were applicable and there was not any modification.

- According to the results of the pilot study subjects included in to the study.

- The Reliability was done on tools by Cronback's Alpha it was (0.95)

Ethical consideration:

- An approval was obtained from the local ethical committee and the study was followed the common ethical principles in clinical research.

- Informed consent was obtained from each patient or from the responsible person for the unconscious patients.

- The investigator was emphasized that the participation is voluntary and the confidentiality and anonymity of the subjects was assured through coding the data.

- Subjects were assured that can they withdraw from the study at any time without any rational at any time.

- There is no risk for study subject during application of the research.

- The study was followed common ethical principles in the clinical research.

\section{Implementation phase}

- The data were collected seven days of the week until patient discharge from intensive care unit (ICU), during period between "April 2015" till "January 2016".

- Control group: The control group subjects were receiving the routine hospital care and evaluated in the same way as the study group subjects. The researcher observed the control group and the routine care the nurse gave it to them.

- Study group: each patient of the study group subjects was exposed to the following interventions in addition to the routine hospital care:-

- Each patient of the study group subjects was received two sessions/day of rehabilitation program (one session in the morning and the other in the evening). Until discharge from ICU according to physician prescription and his condition on daily bases. Each session was taken about 30-45 minute not include time for fulfilling each tool (first tool took about 20 minute and second tool took 10 minutes)

- Patient level of conscious was assessed before session then chest physiotherapy (postural drainage, vibration and percussion), Manual hyperinflation using manual ambo bag followed by suction was performed.

- After finishing chest physiotherapy , physical physiotherapy is applied and progression of activities was dependent on patient tolerance and stability, level of conscious and ability to follow command

- During physical therapy and once patient was able to cooperate and express his feeling, Borg rate of perceived exertion was used to evaluate the patient's perceived fatigue level during the intervention This used only for study group

- Heart rate, respiration, blood pressures, mean arterial pressure (MAP), and saturation reading from pulse oximetry for patient were monitored 3 times/session

- Temperature, blood gases values and CVP readings were evaluated 2 times / session.

- Parameters of mechanical ventilator were evaluated once/ session before application of rehabilitation 
program. control group subjects was evaluated in the same way as the study group

- Each complication were reviewed, the occurrence of a complication was counted only once. Adverse events during mobility sessions as: self-extubation, decannulation, removal of bladder catheter, Hemodynamic unstable, and were also reviewed.

- By the end of the session leaving the patient to the routine nursing interventions

\section{Statistical design $S$}

All data were recorded in a special chart for every patient. The collected data were coded, analyzed and tabulated .Data entry and analysis were done using SPSS 19.0 statistical software package. Data were presented using descriptive statistics in the form of frequencies and percentages for qualitative variables, and means and standard deviations for quantitative variables. Quantitative continuous data were compared using analysis of variance test in case of comparisons between two independent groups. Using independent T-test and chi-square test to determine significant, it is considered significant when $\mathrm{P} \leq 0.05$ significant and non- significant when $\mathrm{P}>0.05$.

\section{Results}

Table (1): Comparison between the study \& control groups as regard socio-demographic $(\mathrm{n}=60)$

\begin{tabular}{|c|c|c|c|c|c|}
\hline \multirow[t]{2}{*}{ Variables } & \multicolumn{2}{|c|}{$\begin{array}{c}\text { Study Group } \\
(\mathbf{n}=\mathbf{3 0})\end{array}$} & \multicolumn{2}{|c|}{$\begin{array}{c}\text { Control Group } \\
(\mathbf{n}=\mathbf{3 0})\end{array}$} & \multirow[t]{2}{*}{ P-value } \\
\hline & No. & $\%$ & No. & $\%$ & \\
\hline \multicolumn{5}{|l|}{ Age } & \multirow{3}{*}{0.196} \\
\hline$<50$ years & 17 & 56.7 & 12 & 40.0 & \\
\hline$\geq 50$ years & 13 & 43.3 & 18 & 60.0 & \\
\hline Mean \pm SD & \multicolumn{2}{|c|}{$46.97 \pm 11.47$} & \multicolumn{2}{|c|}{$52.20 \pm 9.66$} & \\
\hline \multicolumn{5}{|l|}{ Sex } & \multirow{3}{*}{$0.004^{*}$} \\
\hline Male & 17 & 56.7 & 27 & 90.0 & \\
\hline Female & 13 & 43.3 & 3 & 10.0 & \\
\hline
\end{tabular}

Table (2) Comparison between the study \& control groups as regard clinical data $(\mathrm{n}=60)$

\begin{tabular}{|c|c|c|c|c|}
\hline \multirow[t]{2}{*}{ Variables } & \multicolumn{2}{|c|}{$\begin{array}{l}\text { Study Group } \\
\quad(n=\mathbf{3 0})\end{array}$} & \multicolumn{2}{|c|}{$\begin{array}{c}\text { Control Group } \\
(n=30)\end{array}$} \\
\hline & No. & $\%$ & No. & $\%$ \\
\hline \multicolumn{5}{|l|}{ Medical diagnosis } \\
\hline Acute renal failure & 3 & 10.0 & 1 & 3.3 \\
\hline COPD & 10 & 33.3 & 13 & 43.3 \\
\hline Pneumonia & 5 & 16.7 & 4 & 13.3 \\
\hline Asthma & 1 & 3.3 & 1 & 3.3 \\
\hline Lung fibrosis & 1 & 3.3 & 0 & 0.0 \\
\hline Acute respiratory failure & 6 & 20.0 & 7 & 23.3 \\
\hline Poisoning & 1 & 3.3 & 1 & 3.3 \\
\hline Postoperative & 3 & 10.0 & 3 & 10.0 \\
\hline \multicolumn{5}{|l|}{ Past medical history } \\
\hline Diabetes mellitus & 2 & 6.7 & 0 & 0.0 \\
\hline Hypertension & 3 & 10.0 & 3 & 10.0 \\
\hline Cancer & 3 & 10.0 & 0 & 0.0 \\
\hline Kidney disease & 4 & 13.3 & 1 & 3.3 \\
\hline COPD & 8 & 26.7 & 10 & 33.3 \\
\hline Respiratory disease & 4 & 13.3 & 9 & 30 \\
\hline No-medical history & 6 & 20 & 7 & 23.3 \\
\hline
\end{tabular}


Table (3): Comparison between the study \& control groups as regard hemodynamic parameters (baseline data) $(n=60)$.

\begin{tabular}{|c|c|c|c|}
\hline \multirow[t]{2}{*}{ Hemodynamic parameters } & Study Group $(\mathbf{n}=\mathbf{3 0})$ & $\begin{array}{c}\text { Control Group } \\
(n=30)\end{array}$ & \multirow[t]{2}{*}{ P-value } \\
\hline & Mean \pm SD & Mean \pm SD & \\
\hline \multicolumn{4}{|l|}{ Heart rate (HR) } \\
\hline Baseline intervention & $99.33 \pm 16.77$ & $101.00 \pm 12.33$ & 0.457 \\
\hline After 10 min of intervention & $103.00 \pm 14.71$ & $104.67 \pm 12.58$ & 0.949 \\
\hline After 30 min of intervention & $100.25 \pm 12.20$ & $101.11 \pm 12.35$ & 0.966 \\
\hline \multicolumn{4}{|l|}{ Systolic BP } \\
\hline Baseline intervention & $120.92 \pm 15.54$ & $114.67 \pm 6.71$ & 0.111 \\
\hline After 10 min of intervention & $122.25 \pm 11.70$ & $117.44 \pm 5.69$ & 0.275 \\
\hline After $30 \mathrm{~min}$ of intervention & $122.50 \pm 10.46$ & $116.89 \pm 5.05$ & 0.086 \\
\hline \multicolumn{4}{|l|}{ Diastolic BP } \\
\hline Baseline intervention & $78.67 \pm 10.53$ & $76.78 \pm 7.64$ & 0.698 \\
\hline After 10 min of intervention & $80.42 \pm 7.03$ & $77.83 \pm 6.71$ & 0.431 \\
\hline After $30 \mathrm{~min}$ of intervention & $80.33 \pm 6.27$ & $78.61 \pm 6.78$ & 0.610 \\
\hline \multicolumn{4}{|l|}{ MAP } \\
\hline Baseline intervention & $91.33 \pm 10.57$ & $88.22 \pm 5.53$ & 0.371 \\
\hline After 10 min of intervention & $94.00 \pm 7.79$ & $89.83 \pm 4.96$ & 0.137 \\
\hline After 30 min of intervention & $94.17 \pm 6.99$ & $89.17 \pm 4.46$ & 0.058 \\
\hline \multicolumn{4}{|l|}{ CVP } \\
\hline Baseline intervention & $11.33 \pm 3.68$ & $10.00 \pm 1.93$ & 0.360 \\
\hline After intervention & $11.08 \pm 4.74$ & $10.06 \pm 1.53$ & 0.888 \\
\hline \multicolumn{4}{|l|}{ Temperature } \\
\hline Baseline intervention & $36.99 \pm 0.58$ & $37.29 \pm 0.72$ & 0.543 \\
\hline After 1 hour intervention & $37.19 \pm 0.65$ & $37.37 \pm 0.56$ & 0.503 \\
\hline \multicolumn{4}{|l|}{ Pulse oximetry } \\
\hline Baseline intervention & $89.50 \pm 4.96$ & $87.56 \pm 4.03$ & 0.360 \\
\hline After 10 min of intervention & $87.92 \pm 2.81$ & $87.61 \pm 2.70$ & 0.684 \\
\hline After 30 min of intervention & $88.42 \pm 2.39$ & $88.72 \pm 2.80$ & 0.898 \\
\hline
\end{tabular}
Chi-square test
$P>0.05$ non significant
$* P<0.05$ significant

Table (4): Comparison between the study \& control groups as regard hemodynamic parameters (at discharge) $(\mathrm{n}=60)$.

\begin{tabular}{|c|c|c|c|}
\hline \multirow[b]{2}{*}{ Hemodynamic parameters } & Study Group $(\mathrm{n}=30)$ & Control Group $(n=30)$ & \multirow[b]{2}{*}{ P-value } \\
\hline & Mean \pm SD & Mean \pm SD & \\
\hline \multicolumn{4}{|l|}{ Heart rate (HR) } \\
\hline Baseline intervention & $96.17 \pm 8.38$ & $109.22 \pm 18.14$ & 0.053 \\
\hline After 10 min of intervention & $112.83 \pm 18.05$ & $99.50 \pm 7.08$ & $0.042 *$ \\
\hline After 30 min of intervention & $97.75 \pm 6.18$ & $106.94 \pm 18.30$ & 0.270 \\
\hline \multicolumn{4}{|l|}{ Systolic BP } \\
\hline Baseline intervention & $115.00 \pm 6.52$ & $117.00 \pm 9.55$ & 0.677 \\
\hline After 10 min of intervention & $115.00 \pm 7.62$ & $117.33 \pm 7.82$ & 0.493 \\
\hline After 30 min of intervention & $116.83 \pm 6.45$ & $117.44 \pm 8.20$ & 0.844 \\
\hline \multicolumn{4}{|l|}{ Diastolic BP } \\
\hline Baseline intervention & $79.25 \pm 7.03$ & $79.94 \pm 7.73$ & 0.932 \\
\hline After 10 min of intervention & $81.58 \pm 5.11$ & $78.96 \pm 7.19$ & 0.173 \\
\hline After 30 min of intervention & $80.67 \pm 6.64$ & $77.28 \pm 7.51$ & 0.116 \\
\hline
\end{tabular}




\begin{tabular}{|c|c|c|c|}
\hline \multirow[b]{2}{*}{ Hemodynamic parameters } & Study Group (n=30) & Control Group $(\mathbf{n}=30)$ & \multirow[b]{2}{*}{ P-value } \\
\hline & Mean \pm SD & Mean \pm SD & \\
\hline \multicolumn{4}{|l|}{ MAP } \\
\hline Baseline intervention & $91.08 \pm 4.94$ & $91.06 \pm 6.44$ & 0.918 \\
\hline After 10 min of intervention & $91.50 \pm 3.26$ & $90.61 \pm 6.16$ & 0.365 \\
\hline After 30 min of intervention & $92.00 \pm 3.36$ & $90.44 \pm 5.66$ & 0.185 \\
\hline \multicolumn{4}{|l|}{ CVP } \\
\hline Baseline intervention & $10.00 \pm 1.41$ & $10.18 \pm 2.35$ & 0.982 \\
\hline After intervention & $9.75 \pm 1.48$ & $10.24 \pm 1.92$ & 0.540 \\
\hline \multicolumn{4}{|l|}{ Temperature } \\
\hline Baseline intervention & $37.14 \pm 0.33$ & $37.62 \pm 0.90$ & 0.081 \\
\hline After 1 hour intervention & $37.06 \pm 0.25$ & $37.59 \pm 0.69$ & 0.012* \\
\hline \multicolumn{4}{|l|}{ Pulse oximetry } \\
\hline Baseline intervention & $93.17 \pm 3.41$ & $90.94 \pm 3.26$ & 0.076 \\
\hline After 10 min of intervention & $92.50 \pm 3.37$ & $90.56 \pm 3.57$ & 0.055 \\
\hline After 30 min of intervention & $97.58 \pm 3.09$ & $93.83 \pm 2.62$ & 0.008* \\
\hline
\end{tabular}

Table (5): Comparison between the study \& control groups as regard arterial blood gases (baseline data) $(\mathbf{n}=60)$

\begin{tabular}{|c|c|c|c|}
\hline \multirow{2}{*}{ Arterial blood gases } & Study Group $(n=30)$ & Control Group $(n=30)$ & \multirow{2}{*}{ P-value } \\
\hline & Mean \pm SD & Mean \pm SD & \\
\hline \multicolumn{4}{|l|}{ PH } \\
\hline Baseline intervention & $7.30 \pm 0.10$ & $7.31 \pm 0.10$ & 0.655 \\
\hline After intervention & $7.32 \pm 0.08$ & $7.32 \pm 0.09$ & 0.781 \\
\hline \multicolumn{4}{|l|}{$\mathrm{PaO}_{2}$} \\
\hline Baseline intervention & $71.91 \pm 16.91$ & $73.03 \pm 19.82$ & 0.932 \\
\hline After intervention & $76.13 \pm 14.57$ & $77.49 \pm 16.91$ & 0.932 \\
\hline \multicolumn{4}{|l|}{$\mathrm{PaCO}_{2}$} \\
\hline Baseline intervention & $69.18 \pm 22.10$ & $67.66 \pm 22.52$ & 0.915 \\
\hline After intervention & $67.69 \pm 20.26$ & $65.24 \pm 21.05$ & 0.783 \\
\hline \multicolumn{4}{|l|}{$\mathrm{HCO}_{3}$} \\
\hline Baseline intervention & $28.05 \pm 6.71$ & $26.23 \pm 5.46$ & 0.687 \\
\hline After intervention & $27.80 \pm 6.69$ & $27.11 \pm 5.73$ & 0.865 \\
\hline \multicolumn{4}{|l|}{$\mathrm{SaO}_{2}$} \\
\hline Baseline intervention & $85.58 \pm 4.01$ & $87.83 \pm 5.08$ & 0.241 \\
\hline After intervention & $87.00 \pm 3.22$ & $88.50 \pm 4.51$ & 0.415 \\
\hline
\end{tabular}

Chi-square test $\quad P>0.05$ non significant $\quad * P<0.05$ significant

Table (6): Comparison between the study \& control groups as regard arterial blood gases (at discharge) $(\mathbf{n}=60)$

\begin{tabular}{|l|c|c|c|}
\hline \multirow{2}{*}{ Arterial blood gases } & Study Group $(\mathbf{n}=\mathbf{3 0})$ & Control Group $(\mathbf{n}=\mathbf{3 0})$ & \multirow{2}{*}{ P-value } \\
\cline { 2 - 3 } & Mean \pm SD & Mean \pm SD & \\
\hline PH & $7.39 \pm 0.04$ & $7.42 \pm 0.10$ & 0.365 \\
\hline Baseline intervention & $7.40 \pm 0.04$ & $7.47 \pm 0.09$ & $\mathbf{0 . 0 1 5}^{*}$ \\
\hline After intervention & $100.68 \pm 8.56$ & $92.65 \pm 12.89$ & 0.211 \\
\hline PaO $\mathbf{P}_{\mathbf{2}}$ & $112.84 \pm 8.44$ & $92.81 \pm 12.82$ & $\mathbf{0 . 0 0 0}$ \\
\hline Baseline intervention & \multicolumn{3}{|c|}{} \\
\hline After intervention &
\end{tabular}




\begin{tabular}{|c|c|c|c|}
\hline \multirow{2}{*}{ Arterial blood gases } & Study Group $(n=30)$ & Control Group $(n=30)$ & \multirow{2}{*}{ P-value } \\
\hline & Mean \pm SD & Mean \pm SD & \\
\hline \multicolumn{4}{|l|}{$\mathrm{PaCO}_{2}$} \\
\hline Baseline intervention & $46.32 \pm 9.42$ & $50.86 \pm 9.94$ & 0.259 \\
\hline After intervention & $46.61 \pm 8.83$ & $49.26 \pm 17.45$ & $0.032 *$ \\
\hline \multicolumn{4}{|l|}{$\mathrm{HCO}_{3}$} \\
\hline Baseline intervention & $23.28 \pm 2.93$ & $23.85 \pm 2.84$ & 0.525 \\
\hline After intervention & $22.83 \pm 2.62$ & $23.61 \pm 2.54$ & 0.384 \\
\hline \multicolumn{4}{|l|}{$\mathrm{SaO}_{2}$} \\
\hline Baseline intervention & $97.83 \pm 2.92$ & $95.33 \pm 4.55$ & 0.126 \\
\hline After intervention & $97.75 \pm 2.70$ & $94.06 \pm 4.54$ & $0.033^{*}$ \\
\hline
\end{tabular}

\section{Chi-square test $\quad P>0.05$ non significant $\quad * P<0.05$ significant}

Table (7): Comparison between the study \& control groups as regard mechanical ventilator parameters_(n=60).

\begin{tabular}{|c|c|c|c|}
\hline \multirow{2}{*}{$\begin{array}{c}\text { Day / mechanical ventilator } \\
\text { parameters }\end{array}$} & Study Group $(n=30)$ & Control Group $(n=30)$ & \multirow{2}{*}{ P-value } \\
\hline & Mean \pm SD & Mean \pm SD & \\
\hline \multicolumn{4}{|l|}{ On admission } \\
\hline Tidal volume & $431.75 \pm 28.49$ & $437.50 \pm 24.66$ & 0.372 \\
\hline PEEP & $6.42 \pm 1.17$ & $7.00 \pm 0.84$ & 0.145 \\
\hline Pressure support & $11.92 \pm 1.38$ & $12.22 \pm 1.06$ & 0.533 \\
\hline Lung compliance & $47.50 \pm 3.53$ & $48.44 \pm 3.54$ & 0.578 \\
\hline PIP & $26.25 \pm 1.55$ & $26.06 \pm 1.39$ & 0.549 \\
\hline $\mathrm{FIO}_{2}$ & $70.94 \pm 9.05$ & $69.03 \pm 14.22$ & 0.578 \\
\hline $\mathrm{PAO}_{2} / \mathrm{FIO}_{2}$ & $293.33 \pm 27.79$ & $273.72 \pm 37.82$ & 0.234 \\
\hline \multicolumn{4}{|l|}{ Last day of weaning } \\
\hline Tidal volume & $507.17 \pm 25.55$ & $435.39 \pm 35.59$ & 0.000* \\
\hline PEEP & $5.48 \pm 0.29$ & $5.78 \pm 0.65$ & 0.112 \\
\hline Pressure support & $8.83 \pm 1.12$ & $10.00 \pm 1.37$ & 0.023* \\
\hline Lung compliance & $59.33 \pm 4.38$ & $56.06 \pm 4.66$ & 0.041* \\
\hline PIP & $24.50 \pm 0.52$ & $26.00 \pm 1.41$ & 0.001* \\
\hline FIO2 & $37.94 \pm 9.08$ & $45.21 \pm 10.01$ & $0.012 *$ \\
\hline $\mathrm{PAO} 2 / \mathrm{FIO} 2$ & $308.50 \pm 29.14$ & $287.44 \pm 23.02$ & $0.001 *$ \\
\hline
\end{tabular}

Chi-square test $\quad P>0.05$ non significant $\quad * P<0.05$ significant

Table (8): Comparison between both study \& control groups in relation to respiratory assessment for (Breathing sounds) $(\mathrm{n}=60)$.

\begin{tabular}{|c|c|c|c|c|c|}
\hline \multirow{2}{*}{ Day / Breathing sounds } & \multicolumn{2}{|c|}{ Study Group $(n=30)$} & \multicolumn{2}{|c|}{ Control Group $(n=30)$} & \multirow{2}{*}{$\begin{array}{c}\text { Significance } \\
\text { test }\end{array}$} \\
\hline & No. & $\%$ & No. & $\%$ & \\
\hline \multicolumn{6}{|l|}{ On Admission } \\
\hline Normal & 9 & 30.0 & 9 & 30.0 & \multirow{5}{*}{0.445} \\
\hline Wheezing & 15 & 50.0 & 16 & 53.3 & \\
\hline Crepitation & 3 & 10.0 & 2 & 6.7 & \\
\hline Crackle & 3 & 10.0 & 2 & 6.7 & \\
\hline Rhonchi & 0 & 0 & 1 & 3.33 & \\
\hline \multicolumn{6}{|l|}{ At $1^{\text {st }}$ Day Of Weaning } \\
\hline Normal & 25 & 83.3 & 19 & 63.3 & \multirow{5}{*}{0.175} \\
\hline Wheezing & 5 & 16.7 & 10 & 33.3 & \\
\hline Crepitation & 0 & 0.0 & 1 & 3.3 & \\
\hline Crackle & 0 & 0.0 & 0 & 0.0 & \\
\hline Rhonchi & 0 & 0.0 & 0 & 0.0 & \\
\hline
\end{tabular}




\begin{tabular}{|c|c|c|c|c|c|}
\hline \multirow{2}{*}{ Day / Breathing sounds } & \multicolumn{2}{|c|}{ Study Group $(n=30)$} & \multicolumn{2}{|c|}{ Control Group $(n=30)$} & \multirow{2}{*}{$\begin{array}{c}\text { Significance } \\
\text { test }\end{array}$} \\
\hline & No. & $\%$ & No. & $\%$ & \\
\hline \multicolumn{6}{|l|}{ At Discharge } \\
\hline Normal & 30 & 100.0 & 16 & 53.3 & \multirow{5}{*}{$0.000 *$} \\
\hline Wheezing & 0 & 0.0 & 13 & 43.3 & \\
\hline Crepitation & 0 & 0.0 & 1 & 3.3 & \\
\hline Crackle & 0 & 0.0 & 0 & 0.0 & \\
\hline Rhonchi & 0 & 0.0 & 0 & 0.0 & \\
\hline
\end{tabular}

Independent t test

$P>0.05$ non significant

$* P<0.05$ significant

Table (9): Comparison between both study \& control group in relation to respiratory assessment for (color of bronchial secretion) $(\mathbf{n}=60)$

\begin{tabular}{|c|c|c|c|c|c|c|c|c|c|c|c|c|c|}
\hline \multirow{3}{*}{$\begin{array}{c}\text { Secretion } \\
\text { Days }\end{array}$} & \multicolumn{4}{|c|}{ Clear } & \multicolumn{4}{|c|}{ Yellowish } & \multicolumn{4}{|c|}{ Greenish } & \multirow{3}{*}{$\begin{array}{c}\text { Significance } \\
\text { test }\end{array}$} \\
\hline & \multicolumn{2}{|c|}{\begin{tabular}{l|} 
Study \\
Group \\
$(\mathbf{n}=\mathbf{3 0})$
\end{tabular}} & \multicolumn{2}{|c|}{$\begin{array}{c}\text { Control } \\
\text { Group } \\
(\mathbf{n}=30)\end{array}$} & \multicolumn{2}{|c|}{$\begin{array}{l}\text { Study } \\
\text { group } \\
(\mathbf{n}=30)\end{array}$} & \multicolumn{2}{|c|}{\begin{tabular}{c|c|} 
Control \\
$\operatorname{group}(n=30)$ \\
\end{tabular}} & \multicolumn{2}{|c|}{$\begin{array}{c}\text { Study } \\
\operatorname{group}(n=30)\end{array}$} & \multicolumn{2}{|c|}{$\begin{array}{c}\begin{array}{c}\text { Control } \\
\operatorname{group}(n=30)\end{array} \\
\end{array}$} & \\
\hline & No. & $\%$ & No. & $\%$ & No. & $\%$ & No. & $\%$ & No. & $\%$ & No. & $\%$ & \\
\hline On admission & 29 & 96.7 & 25 & 82.3 & 1 & 3.3 & 5 & 16.6 & 0 & 0 & 0 & 0 & $\mathrm{P}=0.402$ \\
\hline $\begin{array}{l}1^{\text {st }} \text { day of } \\
\text { weaning }\end{array}$ & 24 & 80.0 & 23 & 76.7 & 3 & 10.0 & 6 & 20.0 & 3 & 10.0 & 1 & 3.3 & $\mathrm{P}=0.103$ \\
\hline At discharge & 30 & 100.0 & 10 & 33.3 & 0 & 0 & 15 & 50.0 & 0 & 0 & 5 & 16.7 & $\mathrm{P}=0.001^{*}$ \\
\hline
\end{tabular}

Table (10): Comparison between both study \& control groups in relation to respiratory assessment for (amount of Bronchial Secretion) (n=60).

\begin{tabular}{|c|c|c|c|c|c|c|c|c|c|c|c|c|c|}
\hline \multirow{3}{*}{$\begin{array}{c}\text { Secretion } \\
\text { Days }\end{array}$} & \multicolumn{4}{|c|}{ Small } & \multicolumn{4}{|c|}{ Moderate } & \multicolumn{4}{|c|}{$\begin{array}{c}\text { Large } \\
\end{array}$} & \multirow{3}{*}{$\begin{array}{c}\text { Significance } \\
\text { test }\end{array}$} \\
\hline & \multicolumn{2}{|c|}{$\begin{array}{l}\text { Study } \\
\text { Group } \\
(\mathbf{n}=30)\end{array}$} & \multicolumn{2}{|c|}{$\begin{array}{c}\text { Control } \\
\text { Group } \\
(\mathbf{n}=30)\end{array}$} & \multicolumn{2}{|c|}{$\begin{array}{c}\text { Study group } \\
(\mathrm{n}=30)\end{array}$} & \multicolumn{2}{|c|}{$\begin{array}{c}\text { Control } \\
\text { Group } \\
(\mathbf{n}=\mathbf{3 0})\end{array}$} & \multicolumn{2}{|c|}{$\begin{array}{c}\text { Study } \\
\text { group } \\
(\mathbf{n}=30)\end{array}$} & \multicolumn{2}{|c|}{$\begin{array}{c}\text { Control } \\
\text { Group } \\
(\mathbf{n}=30)\end{array}$} & \\
\hline & No. & $\%$ & No. & $\%$ & No. & $\%$ & No. & $\%$ & No. & $\%$ & No. & $\%$ & \\
\hline & 19 & 63.3 & 12 & 400 & 7 & & 16 & & 4 & 121 & 0 & & \\
\hline $\begin{array}{l}1^{\text {st }} \text { day of } \\
\text { weaning }\end{array}$ & 10 & 33.3 & 7 & 23.3 & 8 & 26.6 & 13 & 43.3 & 12 & 40.0 & 10 & 33.3 & $\mathrm{P}=0.241$ \\
\hline At discharge & 22 & 73.3 & 13 & 43.3 & 4 & 13.3 & 12 & 40.0 & 4 & 13.3 & 5 & 16.6 & $\mathrm{P}=0.009 *$ \\
\hline
\end{tabular}

Independent t test $\quad P>0.05$ non significant $\quad * P<0.05$ significant

Table (11): Comparison between both study \& control group in relation to respiratory assessment for (viscosity of Bronchial Secretion).

\begin{tabular}{|c|c|c|c|c|c|c|c|c|c|}
\hline \multirow{3}{*}{$\begin{array}{c}\text { Secretion } \\
\text { Days }\end{array}$} & \multicolumn{4}{|c|}{ Loose } & \multicolumn{4}{|c|}{ Viscid } & \multirow{3}{*}{$\begin{array}{c}\text { Significance } \\
\text { test }\end{array}$} \\
\hline & \multicolumn{2}{|c|}{\begin{tabular}{c|c} 
Study \\
Group $(\mathbf{n}=30)$
\end{tabular}} & \multicolumn{2}{|c|}{$\begin{array}{c}\text { Control } \\
\text { Group }(n=30) \\
\end{array}$} & \multicolumn{2}{|c|}{$\begin{array}{c}\text { Study group } \\
(\mathbf{n}=30)\end{array}$} & \multicolumn{2}{|c|}{$\begin{array}{c}\text { Control } \\
\operatorname{group}(n=30)\end{array}$} & \\
\hline & $\mathbf{N}$ & $\%$ & $\mathbf{N}$ & $\%$ & $\mathbf{N}$ & $\%$ & $\mathbf{N}$ & $\%$ & \\
\hline Om admission & 20 & 66.7 & 19 & 38.9 & 10 & 33.3 & 11 & 36.6 & $\mathrm{P}=0.136$ \\
\hline $1^{\text {st }}$ day of weaning & 22 & 73.3 & 17 & 23.3 & 8 & 16.7 & 13 & 43.3 & $\mathrm{P}=0.031 *$ \\
\hline At discharge & 23 & 76.6 & 20 & 66.7 & 7 & 23.3 & 10 & 33.3 & $\mathrm{P}=0.088$ \\
\hline
\end{tabular}

Independent t test $\quad P>0.05$ non significant $\quad * P<0.05$ significant 
Table (12): Comparison between the study \& control groups as regard weaning variables $(n=60)$

\begin{tabular}{|c|c|c|c|c|c|}
\hline \multirow[t]{2}{*}{ Weaning variables } & \multicolumn{2}{|c|}{$\begin{array}{c}\text { Study Group } \\
(\mathbf{n = 3 0})\end{array}$} & \multicolumn{2}{|c|}{$\begin{array}{l}\text { Control Group } \\
\quad(n=30)\end{array}$} & \multirow[t]{2}{*}{ P-value } \\
\hline & No. & $\%$ & No. & $\%$ & \\
\hline \multicolumn{5}{|c|}{ No. of spontaneous breathing trail } & \multirow{3}{*}{0.152} \\
\hline Once & 27 & 90.0 & 21 & 70.0 & \\
\hline Twice & 3 & 10.0 & 9 & 30.0 & \\
\hline \multicolumn{5}{|l|}{ Weaning duration } & \multirow{3}{*}{0.257} \\
\hline Mean \pm SD & \multicolumn{2}{|c|}{$9.50 \pm 3.45$} & \multicolumn{2}{|c|}{$10.89 \pm 3.07$} & \\
\hline Range & \multicolumn{2}{|c|}{$8.0-16.0$} & \multicolumn{2}{|c|}{$8.0-19.0$} & \\
\hline \multicolumn{6}{|l|}{ Rapid shallow breathing index } \\
\hline Baseline & \multicolumn{2}{|c|}{$120 \pm 10.09$} & \multicolumn{2}{|c|}{$119 \pm 9.03$} & 0.526 \\
\hline At discharge & \multicolumn{2}{|c|}{$80 \pm 6.4$} & \multicolumn{2}{|c|}{$95 \pm 8.01$} & $0.042 *$ \\
\hline
\end{tabular}

Table (13): Comparison between the study \& control groups as regard complete blood count $(n=60)$

\begin{tabular}{|c|c|c|c|}
\hline \multirow{2}{*}{$\begin{array}{c}\text { Days /complete blood } \\
\text { count }\end{array}$} & $\begin{array}{c}\text { Study Group } \\
(\mathbf{n}=\mathbf{3 0})\end{array}$ & $\begin{array}{l}\text { Control Group } \\
(\mathbf{n}=\mathbf{3 0})\end{array}$ & \multirow[t]{2}{*}{ P-value } \\
\hline & Mean \pm SD & Mean \pm SD & \\
\hline \multicolumn{4}{|l|}{ On admission } \\
\hline WBC & $8.06 \pm 2.08$ & $8.29 \pm 1.57$ & 0.783 \\
\hline $\mathrm{RBC}$ & $4.03 \pm 0.52$ & $4.05 \pm 0.68$ & 0.483 \\
\hline Hemoglobin & $12.92 \pm 1.55$ & $13.03 \pm 2.06$ & 0.611 \\
\hline Hematocrit & $39.04 \pm 8.12$ & $33.36 \pm 6.13$ & 0.098 \\
\hline PLT & $237.72 \pm 47.99$ & $228.08 \pm 33.33$ & 0.899 \\
\hline \multicolumn{4}{|l|}{$1^{\text {st }}$ day of weaning } \\
\hline WBC & $9.08 \pm 1.93$ & $10.82 \pm 3.50$ & 0.299 \\
\hline RBC & $3.95 \pm 0.50$ & $3.96 \pm 0.75$ & 0.595 \\
\hline Hemoglobin & $13.15 \pm 1.39$ & $12.81 \pm 2.50$ & 0.882 \\
\hline Hematocrit & $39.15 \pm 8.06$ & $34.00 \pm 6.44$ & 0.098 \\
\hline PLT & $246.00 \pm 43.89$ & $222.17 \pm 28.84$ & 0.243 \\
\hline \multicolumn{4}{|l|}{ At discharge } \\
\hline WBC & $9.20 \pm 1.49$ & $11.99 \pm 3.46$ & $0.025 *$ \\
\hline $\mathrm{RBC}$ & $3.95 \pm 0.56$ & $3.87 \pm 0.74$ & 0.915 \\
\hline Hemoglobin & $12.98 \pm 0.85$ & $12.58 \pm 2.52$ & 0.611 \\
\hline Hematocrit & $39.34 \pm 6.23$ & $36.50 \pm 6.27$ & 0.103 \\
\hline PLT & $246.17 \pm 45.85$ & $222.25 \pm 24.48$ & 0.149 \\
\hline
\end{tabular}

Chi-square test $P>0.05$ non significant $\quad * P<0.05$ significant

Table (14): Comparison between the study \& control groups as regard liver and renal function $(n=60)$

\begin{tabular}{|c|c|c|c|}
\hline \multirow{2}{*}{$\begin{array}{l}\text { days / Liver and } \\
\text { renal function }\end{array}$} & Study Group $(n=30)$ & Control Group $(n=30)$ & \multirow{2}{*}{ P-value } \\
\hline & Mean \pm SD & Mean \pm SD & \\
\hline \multicolumn{4}{|l|}{ On admission } \\
\hline B. urea & $12.74 \pm 6.29$ & $14.88 \pm 6.90$ & 0.290 \\
\hline Creatinine & $1.55 \pm 1.98$ & $1.20 \pm 0.78$ & 0.949 \\
\hline T. bilirubin & $1.34 \pm 0.63$ & $1.19 \pm 0.42$ & 0.419 \\
\hline T. protein & $4.06 \pm 1.02$ & $5.00 \pm 0.61$ & $0.001 *$ \\
\hline Albumin & $3.19 \pm 1.25$ & $4.13 \pm 0.82$ & $0.007 *$ \\
\hline
\end{tabular}




\begin{tabular}{|c|c|c|c|}
\hline \multirow{2}{*}{$\begin{array}{l}\text { days / Liver and } \\
\text { renal function }\end{array}$} & Study Group $(n=30)$ & Control Group $(n=30)$ & \multirow{2}{*}{ P-value } \\
\hline & Mean \pm SD & Mean \pm SD & \\
\hline \multicolumn{4}{|l|}{$1^{\text {st }}$ day of weaning } \\
\hline B. urea & $13.19 \pm 5.64$ & $16.64 \pm 8.69$ & 0.162 \\
\hline Creatinine & $0.94 \pm 0.47$ & $1.44 \pm 1.11$ & 0.195 \\
\hline T. bilirubin & $1.32 \pm 0.63$ & $1.28 \pm 0.44$ & 0.848 \\
\hline T. protein & $4.07 \pm 1.04$ & $4.94 \pm 0.59$ & $0.004 *$ \\
\hline Albumin & $3.21 \pm 1.30$ & $4.14 \pm 0.81$ & 0.009* \\
\hline \multicolumn{4}{|l|}{ At discharge } \\
\hline B. urea & $12.96 \pm 5.51$ & $15.75 \pm 7.22$ & 0.138 \\
\hline Creatinine & $0.92 \pm 0.44$ & $1.27 \pm 0.91$ & 0.259 \\
\hline T. bilirubin & $1.37 \pm 0.63$ & $1.26 \pm 0.45$ & 0.537 \\
\hline T. protein & $4.08 \pm 1.05$ & $5.01 \pm 0.57$ & 0.003* \\
\hline Albumin & $3.22 \pm 1.30$ & $4.05 \pm 0.77$ & $0.032 *$ \\
\hline
\end{tabular}

Chi-square test $P>0.05$ non significant $\quad * P<0.05$ significant

Table (15): Comparison between the study \& control groups as regard serum electrolytes $(n=60)$

\begin{tabular}{|c|c|c|c|}
\hline \multirow{2}{*}{$\begin{array}{c}\text { Days /serum } \\
\text { electrolytes }\end{array}$} & Study Group $(n=30)$ & Control Group $(n=30)$ & \multirow{2}{*}{ P-value } \\
\hline & Mean \pm SD & Mean \pm SD & \\
\hline \multicolumn{4}{|l|}{ On admission } \\
\hline Sodium & $139.41 \pm 4.72$ & $136.38 \pm 4.94$ & 0.133 \\
\hline Potassium & $4.43 \pm 0.73$ & $4.14 \pm 0.66$ & 0.120 \\
\hline Magnesium & $1.40 \pm 0.50$ & $1.27 \pm 0.37$ & 0.565 \\
\hline Calcium & $8.70 \pm 0.99$ & $8.79 \pm 0.83$ & 0.882 \\
\hline \multicolumn{4}{|l|}{$1 \mathrm{~s}$ day of weaning } \\
\hline Sodium & $141.83 \pm 6.93$ & $140.08 \pm 7.98$ & 0.290 \\
\hline Potassium & $4.45 \pm 0.80$ & $4.08 \pm 0.54$ & 0.067 \\
\hline Magnesium & $1.47 \pm 0.41$ & $1.40 \pm 0.29$ & 0.717 \\
\hline Calcium & $8.68 \pm 0.98$ & $8.78 \pm 0.83$ & 0.865 \\
\hline \multicolumn{4}{|l|}{ At discharge } \\
\hline Sodium & $140.48 \pm 3.46$ & $137.18 \pm 5.26$ & 0.133 \\
\hline Potassium & $4.08 \pm 0.48$ & $4.12 \pm 0.76$ & 0.749 \\
\hline Magnesium & $1.42 \pm 0.43$ & $1.29 \pm 0.34$ & 0.523 \\
\hline Calcium & $8.78 \pm 0.95$ & $8.68 \pm 0.88$ & 0.566 \\
\hline
\end{tabular}

Chi-square test $P>0.05$ non significant $\quad * P<0.05$ significant

Table (16): Comparison between the study \& control groups as regard neurological assessment $(n=60)$

\begin{tabular}{|l|c|c|c|}
\hline \multirow{2}{*}{ Richmond agitation sedation scale } & Study Group $(\mathbf{n}=\mathbf{3 0})$ & Control Group $(\mathbf{n}=\mathbf{3 0})$ & \multirow{2}{*}{ P-value } \\
\cline { 2 - 4 } & Mean \pm SD & Mean \pm SD & 0.814 \\
\hline $\mathbf{1}^{\text {st }}$ day of sedation & $-0.22 \pm 2.05$ & $-0.58 \pm 1.83$ & $0.016^{*}$ \\
\hline Last day of weaning from sedation & $-0.06 \pm 0.24$ & $-0.75 \pm 1.42$ & 0 \\
\hline
\end{tabular}

Chi-square test $P>0.05$ non significant $\quad * P<0.05$ significant

Table (17): Borg scale on admission and discharge in the study group ( $n=30)$

\begin{tabular}{|c|c|c|}
\hline \multicolumn{2}{|c|}{ Study group $(\mathbf{n = 3 0})$} & \multirow{2}{*}{ P-value } \\
\hline On admission & At discharge & \\
\hline Mean \pm SD & Mean \pm SD & $0.001 *$ \\
\hline $0.62 \pm 1.41$ & $5.02 \pm 0.86$ & \\
\hline
\end{tabular}

Chi-square test $P>0.05$ non significant $\quad * P<0.05$ significant 
Table (18): Comparison between the study \& control groups as regard to complications $(\mathrm{n}=60)$

\begin{tabular}{|l|c|c|c|c|c|}
\hline \multirow{2}{*}{ Complications } & \multicolumn{2}{|c|}{ Study Group (n=30) } & \multicolumn{2}{c|}{ Control Group (n= 30) } & \multirow{2}{*}{ P-value } \\
\cline { 2 - 5 } & No. & \% & No. & \% & \\
\hline Related exercise & 2 & 6.7 & 0 & 0.0 & 0.472 \\
\hline Weaning failure & 3 & 10.0 & 11 & 36.6 & $\mathbf{0 . 0 3 8}^{*}$ \\
\hline Deep venous thrombosis & 0 & 0.0 & 2 & 6.7 & 0.472 \\
\hline Ventilator associated pneumonia & 4 & 13.3 & 10 & 33.3 & $\mathbf{0 . 0 4 9}$ \\
\hline Ventilator associated lung injury & 6 & 20.0 & 8 & 26.7 & 0.542 \\
\hline $\begin{array}{l}\text { Progressive acute respiratory distress } \\
\text { syndrome }\end{array}$ & 6 & 20.0 & 11 & 36.7 & 0.373 \\
\hline Muscle contracture & 0 & 0.0 & 4 & 13.3 & 0.121 \\
\hline
\end{tabular}

Independent $t$ test

$P>0.05$ non significant

$* P<0.05$ significant

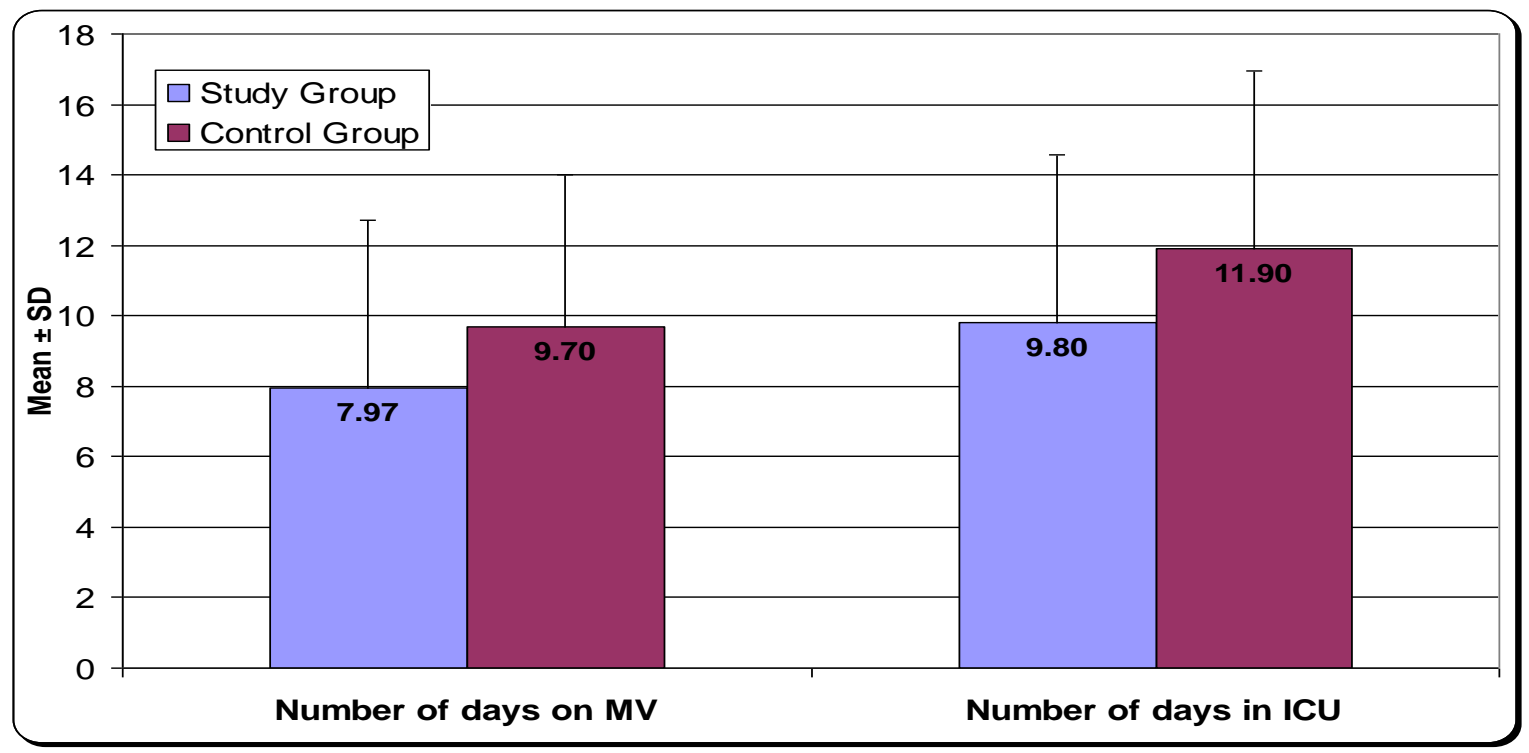

Figure (1):- Comparison between the study and control groups as regard to duration of ventilator dependency \&length of ICU stay $(n=60)$.

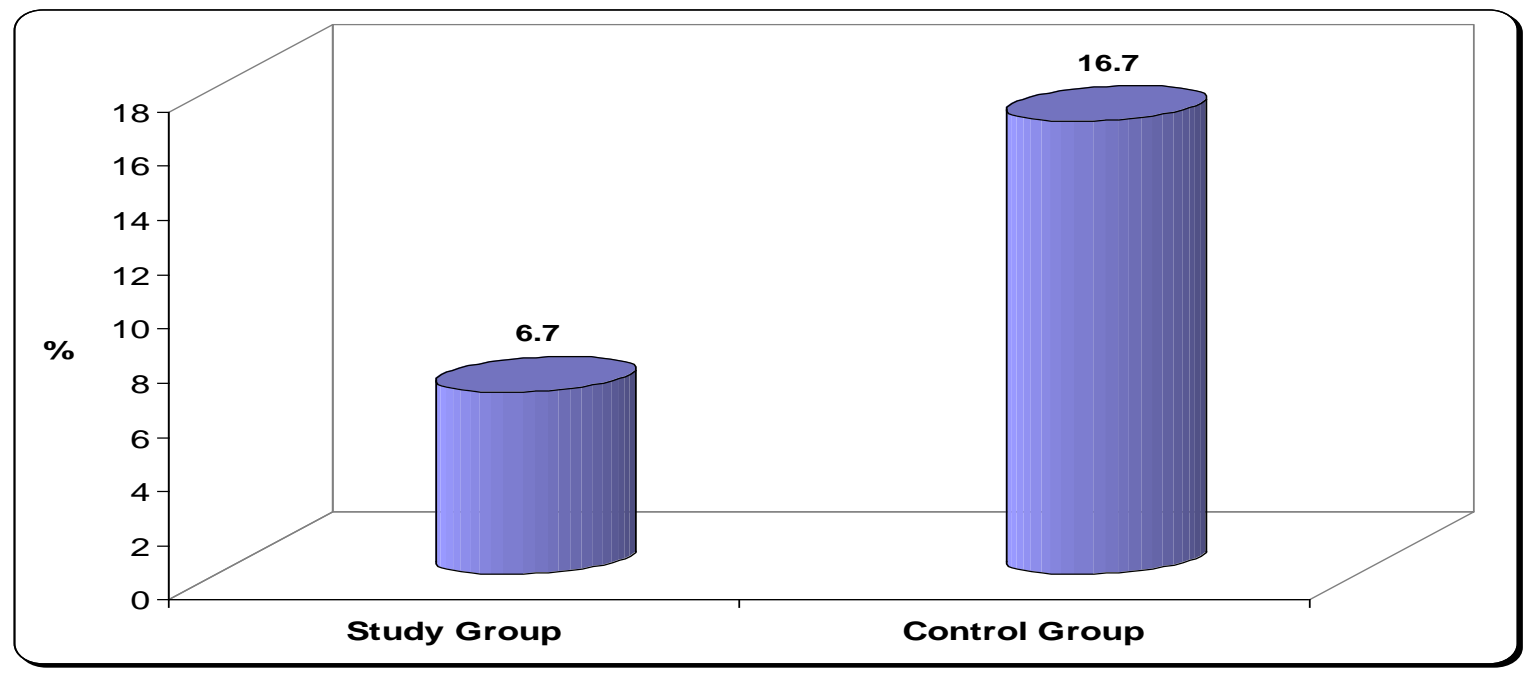

Figure (2): Comparison between the study \& control groups regarded to mortality 
Table (1): Represents comparison between the study and control groups as regard their socio-demographic data. It was found that the mean age in study group was $46.97 \pm 11.47$ years versus $52.20 \pm 9.66$ years in control group with no statistical significant difference $(\mathrm{p}=0.196)$. Also, $56.7 \%$ of the study group were male versus $90.0 \%$ in control group with statistical significant difference $(\mathrm{P}=0.004)$.

Table (2): Represents comparison between the study and control groups as regard their clinical data. It noticed that the most common medical diagnosis was COPD in both study and control groups $(33.3 \%$ vs. $43.3 \%$ respectively); Also, COPD was the common past medical history in both groups (26.7\% vs. $33.3 \%$ respectively).

Tables (3): Illustrate that no statistical significant differences between the two groups on baseline according to hemodynamic parameters (HR, SBP, DBP, MAP, T, CVP and pulse oximetry).

Tables (4): Show the hemodynamic parameters of the studied groups. It was found that there were no statistical significant differences between study and control groups except in heart rate after $10 \mathrm{~min}$ of intervention $(\mathrm{p}=0.042) \&$ pulse oximetry after $30 \mathrm{~min}$ of intervention $(\mathrm{P}=0.008)$ and temperature which was higher in control group than in study group with statistical significant difference $(\mathrm{P}=0.012)$.

Tables (5): Illustrate that there were no statistical significant differences found between the two groups on baseline data in relation to all items arterial blood gases parameters.

Table (6): Demonstrates that there were statistical significant differences between the two groups regarding $\mathrm{PH}, \mathrm{PaO} 2, \mathrm{PaCO} 2$ and $\mathrm{SaO} 2$ after intervention at discharge.

Table (7): Presents mechanical ventilator parameters in study and control groups. This table shows that there were no statistical significance differences between study and control groups. While at last day of weaning, there were statistical significant differences regarding all items of mechanical ventilator parameters except in PEEP.

Table (8): Illustrates that there were statistical significant differences between the two studied groups regarding breathing sound at discharge $(\mathrm{p}=$ 0.000 ), while there were no statistical significant differences between the two studied groups on admission, and first day of weaning.

Table (9): Shows characteristic of both study and control group in relation to respiratory assessment (color of bronchial secretions). It was founded that there were no statistical significant difference between both group on admission and first day of weaning. But there were statistical significant differences $(p=0.001)$ at discharge between both group.
Table (10): Illustrates characteristic of both study and control groups in relation to respiratory assessment (amount of bronchial secretions). It was noted that there were statistical significant differences between the two studied groups regarding amount of bronchial secretions on admission and at discharge $(\mathrm{p}=0.028$ to 0.009 respectively), while there were no statistical significant differences between the two studied groups in first day of weaning

Table (11): Showed characteristic of both study and control group in relation to respiratory assessment (viscosity of bronchial secretions). Shows that more than $(73.3 \%)$ of study group subject had loose secretion versus $(23.3 \%)$ in control group subject with statically significant difference between the both groups ( $\mathrm{p}=0.031$ ) on 1 st day of weaning, and also there were no significance difference during day of admission and at discharge between both group.

Table (12): Demonstrates that there were no statistical significant differences between the two groups regarding weaning data except in rapid shallow breathing index

Table (13): Shows comparison between both study \& control groups as regard complete blood count. It was founded there were statistical significance differences at discharge as regard WBC $(\mathrm{p}=0.025)$

Table (14): Demonstrates that both groups show a slight decreased in mean values of renal function tests but still within normal values. There were founded statistical significance differences between both groups as regard to $\mathrm{T}$. protein and albumin on admission, and $1^{\text {st }}$ day of weaning and at discharge. While there were no statistical significance differences as regard to total bilirubin, urea \& Creatinine in both groups.

Table (15): Shows that there were no statistical significance differences between both groups as regard all items of serum electrolytes.

Table (16): Represents comparison between study and control groups as regard Richmond agitation sedation scale. It was founded that there was no statistical significance difference between both groups on $1^{\text {st }}$ day of sedation. But show that there was statistical significance difference during last day of weaning from sedation $(\mathrm{p}=0.016)$ between both groups.

Table (17): Shows that there were statistical significance differences between day of admission and at discharge as regard to Borg scale in study group.

Table (18): Shows that only two patients of study group developed oxygen desaturation as an adverse effect related to exercise. Also there were no statistical significance differences in all items of complications except weaning failure \& ventilator associated pneumonia between both groups. 
Figure (1): This figure illustrates that the mean value of number of days on MV and number of days in ICU were higher in control group than in the study group. Figure (2): This figure illustrates that mean values of mortality on mechanical ventilation in study and control group were 6.7 vs. 16.7 respectively

\section{Discussion}

Patients who survive critical illness experience reduced health-related quality of life, impaired physical and cognitive functioning as well as psychological morbidity. These changes may be inter-connected and in part may relate to the loss of physical function at the time of critical illness. Rehabilitation of critically ill patients has largely focused on the post-acute and home phases of recovery. However, recent data show that it is safe, feasible and beneficial to engage critically ill patients in rehabilitation activities early on in their illness. (Goddard and Cuthbertson, 2012)

The discussion will cover the main result findings as follows:

Part 1 :-Demographic data \& clinical characteristics of patients

The personal characteristics of the studied patients show no significant difference in age of the rehabilitation and control groups. This result was accepted in the quesi experimental study of The Team Study Investigators (2015). And also randomized controlled trial for Burtin et al., (2009)

Primary disease of the rehabilitation group and control group and past medical history was COPD. This result was agreed to that found by Vitacca et al., (2014) \& Clini et al., (2011) but is opposite to that found by Dong et al., (2014) who reported acute respiratory distress syndrome as the primary cause for admission at ICU.

Part 2: The Effect of early rehabilitation

hemodynamic parameters

In the present study, no significant statistical difference found between the intervention and control groups on day of admission regarding the mean of heart rate, systolic and diastolic blood pressure, mean arterial pressure, temperature and pulse oximetry either before, during or after the rehabilitation. This in agreement with Egerod et al., (2010). who found the baseline characteristics in the two groups was similar.

However the study group showed high mean as compared to the control group subjects during session (after 5 minutes) with significant statistical difference. This supported by the findings of Stiller et al., (2004) \& Bourdin et al., (2010). On the opposite side, Burtin et al., (2009) reported that no changes in heart rate, systolic blood pressure, diastolic blood pressure, or respiratory rate.

Part 3:The Effect of early rehabilitation on Mechanical ventilator parameters \& Arterial blood gases.

Early rehabilitation is practiced routinely have been concerned with appreciating the acute physiological effects increases over baseline ventilation, tidal volume, and respiratory rate in mechanically ventilated patients (Chang et al., 2004).

This study revealed insignificance difference between study and control group on the first day of admission in the all mechanical ventilator parameters except Positive end expiratory pressure (PEEP) which is significantly lower among the study group than the control group. The opposite was found at the discharge, there are significant statistical differences between the two groups regarding all mechanical ventilator parameters except PEEP. The study group had lower $\mathrm{FiO}_{2}$ and higher $\mathrm{PaO}_{2} / \mathrm{FiO}_{2}$ than the control group. Tidal volume, pressure support, lung compliance and peak inspiratory pressure (PIP) which improved than the readings on admission and they are better compared to the control group results.

Effects of chest physiotherapy on various respiratory parameters of the patients under intubation and mechanical ventilation have been well documented by Paratz et al., (2002) which have shown to significantly increase lung compliance (CL ) and $\mathrm{PaO}$ 2 : fio 2 , and decrease PCO 2 of treatment group in a study to determine the effect of manual hyperinflation on hemodynamics, gas exchange and respiratory mechanics in ventilated patients. This is agreed with the study of Moreira et al., (2015) found an increase in dynamic lung compliance, tidal volume, and peripheral oxygen saturation in addition to a reduction of respiratory system, after applying the early respiratory physiotherapy protocol. But disagree with the results of Dong et al., (2014).

Mackenzie, 2011 concluded that chest physiotherapy modalities consisting of (postural drainage, percussion, vibration and suction) has beneficial effect on oxygenation. ware, (2010). added that blood gases improve with the increase in the frequency of number of chest physiotherapy .

Early rehabilitation of mechanically ventilated patients results in improved respiratory muscle strength (Schweickert et al., 2009). The most common change in patient appearance seen during the mobilization treatment was an alteration in respiratory pattern (Stiller et al., 2004).

Part 4: Effect of rehabilitation on laboratory investigation

It was found that there are no satistically significant differences between the intervention group and the control group regarding the findings of complete 
blood count except WBCs was significantly higher in the control group than the study group at discharge time. which is opposite to results of study of Burtin et al., (2009). reported white blood cells were higher among study than control group

The findings of this study show that the mean values of serum electrolytes (sodium, calcium and magnesium) were within normal values on admission, on the first day of weaning and at the discharge time without statistical significant difference between the two groups. This is supported by Khalil et al., (2012). They found that the mean of total serum magnesium, total serum calcium, and serum were $1.6 \mathrm{mg} / \mathrm{dl}$ and $7.6 \mathrm{mg} / \mathrm{dl}$ respectively.

The mean serum albumin which a measure of nutritional status among the intervention group slightly increased on admission but it is significantly lower than the control group. This finding is similar to the result of Clini et al., (2011) as serum albumin of their studied patients on admission to the respiratory intensive care unit was $3.0 \pm 0.3 \mathrm{~g} / \mathrm{dl}$ while higher than that founded by Khalil et al., (2012), who reported that the mean serum albumin of patients with normal nerve conduction .

In this study there was no significance difference between study and control group at 1st day sedation. But there is significance difference in study group compared with control group during last day of weaning from sedation $(\mathrm{p}=0.016)$

The Team Study Investigators (2015) reported that all patients who were mobilized out of bed and stood had a Richmond Agitation and Sedation Scale score of -1 to +1 , while some patients were able to sit over the edge of the bed with a Richmond Agitation and Sedation Scale score of between -2 and +2 .

In this study the mean value of Borg scale at discharge (5.02) which is agree with Denehy et al 2013 which reported that Target Borg Scale score of exercise rehabilitation in ICU was 3 to 5

Part 5: Effect of early rehabilitation on outcome (Complications, LOS on ICU \& NO. of days on MV)

In the current study, there are no significant differences in related to exercise side effects, weaning failure, deep venous thrombosis, ventilator associated pneumonia and ventilator associated lung injury, progressive ARDS, muscle contracture and mortality between both groups. These findings supported by Dong et al., (2014). who reported no serious adverse events occurred in the rehabilitation group, and only one patient developed orthostatic.

Castro et al., (2015). found that patients admitted in study group presented a lower incidence of respiratory infections $(\mathrm{p}=0.004)$ than patients admitted control group. Weaning failure frequently occurred because of inadequate ventilatory drive, respiratory muscle weakness, respiratory muscle fatigue, increased work of breathing or cardiac failure (Epstein, 2002).

Morris et al., (2008). established that more patients received physical therapy during their ICU stay with a trend toward decreased hospital mortality

Length of stay, Duration of mechanical ventilation Early rehabilitation may be less costly through decreased hospital and/or ICU length of stay (LOS) and duration of mechanical ventilation (Adler \& Malone, 2012, Malkoc et al., 2009, Burtin et al., 2009, Morris et al., 2008, Needham et al., 2010).

In the present study, patients in the control group had higher duration connected to mechanical ventilators and longer ICU stay compared to study group who with statistically significant differences. These results supported by the quality improvement project which found an association between early rehabilitation and a decrease in ICU and hospital LOS. In the other side, Campbell (2014), \& Routsi et al., (2010), noted a reduction in number of days of mechanical ventilation, but this difference was not statistically significant.

\section{Conclusion}

Based on the findings of the present study, it can be concluded that:- Rehabilitation should be considered a cornerstone in the comprehensive management of mechanically ventilated patients in the intensive care unit and, when applied early, may benefit patients, prevent some ICU complications, reducing mechanical ventilation support need and, improve outcomes.

\section{Recommendations}

Based on the finding of the current study, the following recommendations are suggested

- Incorporate rehabilitation therapy into treatment plan for mostly critically ill patients.

- Provide educational program about mechanically ventilated care.

- $\quad$ Repeat this research on a large sample size and different governmental hospital for generalization.

- Further researches are recommended to include information about the follow-up of these patients out of ICU.

\section{References}

1. Adler, J., \& Malone, D., (2012): Early mobilization in the intensive care unit: a systematic review. Cardiopulm Phys Ther J, vol 23, pp. 5-13. 
2. Ambrosino, N., \& Makhabah, N., (2014): Physiotherapy in the ICU : for mechanically ventilated patients, early physiotherapy has been shown to improve quality of life and to prevent ICU-associated complications like deconditioning, ventilator dependency, and respiratory conditions." RT for Decision Makers in Respiratory Care. Available at: http://www.rtmagazine.com/2014/07/physiothera py-icu/ . Accessed 26 Dec. 2016.

3. Ambrosino, N., Venturelli, E., Vagheggini, G., \& Clini, E., (2012): Rehabilitation, weaning and physical therapy strategies in the chronic critically ill patients. Eur Respir J, vol 39 pp. 487-492.

4. Avendaño, M., \& Güell, R., (2003): Rehabilitation in Patients with Neuromuscular and Chest Wall Diseases. Archivos de Bronconeumologia, Vol. 39 No.12.

5. Borg, G., (1998): Borg's perceived exertion and pain scales. Champaign: Human Kinetics. Available at: http://www.sciencedirect.com/science/article/pii/S 1836955312700784. Accessed 1 Feb. 2015

6. Bourdin, G., Barbier, J., Burle, J., Durante, G., Passant, S., Vincent, B., Badet, M., Bayle, F., Richard, J., \& Guérin, C., (2010): The feasibility of early physical activity in intensive care unit patients: A prospective observational one-center study. Resp Care, vol 55, pp. 400-407.

7. Burtin, C., Clerckx, B., Robbeets, C., Ferdinande P., Langer, D., Troosters, T., Hermans, G., Decrame, M., \& Gosselink, R., (2009): Early exercise in critically ill patients enhances short-term functional recovery. Crit Care Med, Vol 37 (9), pp. 2499-2505.

8. Campbell, M., (2014): The effect of an early mobility protocol in critically ill mechanically ventilated patients on incidence and duration of delirum and length of stay. Available at: http://corescholar.libraries.wright.edu/nursing_dn p/4. Accessed at 2 Mar. 2016.

9. Carlin, W., (2009): Pulmonary Rehabilitation and Chronic Lung Disease: Opportunities for the Respiratory Therapist. Respiratory care journal, Vol 54 No 8.

10. Castro-Avila, A., Serón, P., Fan, E., Gaete, M., Mickan, S., (2015): Effect of Early Rehabilitation during Intensive Care Unit Stay on Functional Status: Systematic Review and Meta-Analysis. Available at: http://dx.doi.org/10.1371/journal.pone.0130722. Accessed at 4 Mar . 2016.
11. Chang, A., Boots, R., Hodges, P., Thomas, P., Paratz, J., (2004) Standing with the assistance of a tilt table improves minute ventilation in chronic critically ill patients. Arch Phys Med Rehabil, vol 85 (12), pp. 1972-1976.

12. Ciesla, N., (2014) Chest Physical Therapy for Patients in the Intensive care units. Journal of American physical therapy. vol 76, pp. 609-625.

13. Clini, E., Crisafulli, E., Antoni, F., Beneventi, C., Trianni, L., Costi, S., Fabbri, L., \& Nava, S., (2011): Functional Recovery Following Physical Training in tracheotomized and chronically ventilated patients. Respiratory Care, 56 (3), pp. 306- 313.

14. Clini, E., \& Ambrosino, N., (2005): Early physiotherapy in the respiratory intensive care unit. Respiratory Medicine. Vol 99, pp. 10961104.

15. Denehy, L., \& Berney, S., (2006): Physiotherapy in the intensive care unit. Physical Therapy Reviews, Vol 11, pp. 49-56.

16. Denehy, Skinner, E., Edbrooke, L., Haines, K., Warrillow, S., Hawthorne, G., Gough, K., Hoorn, S., Morris, M., \& Berney, S., (2013): Exercise rehabilitation for patients with critical illness: a randomized controlled trial with 12 months of follow-up. Critical Care, 17:R156.

17. Dong, Z., Yu B., Sun, Y., Fang, W., \& Li, L., (2014): Effects of early rehabilitation therapy on patients with mechanical ventilation. World $\mathbf{J}$ Emerg Med, vol 5 (1), pp. 48-52.

18. Egerod, I., Jensen, M., Herling, S., \& Welling, K., (2010): Effect of an analgo-sedation protocol for neurointensive patients: a two-phase interventional non-randomized pilot study. Critical Care, 14: R71 doi: 10.1186/ cc8978.

19. Epstein, S., (2002): Weaning from mechanical ventilation. Respir Care, vol 47, pp. 454-466.

20. Elliot, D., Eitken, L., Chaboyer, W., (2012): ACCCNs Critical Care Nursing. $2^{\text {nd }}$ edition, Chapter 14 respiratory alteration and management, pp. 452-385.

21. Goddard, S., \& Cuthbertson, B., (2012): Rehabilitation and critical illness. Anesthesia and intensive care medicine. Volume 13(5), Pages 214-216.

22. Heffner, E., \& Byock, I., (2002): Palliative and End-of-life Pearls. Philadelphia: Hanley \& Belfus, pp. 76-79. 
23. Hinkle, L., \& Cheever, H., (2014): Brunner\& suddarth's text book of medical surgical nursing, $13^{\text {th }}$ edition, chapter (10) principal and practice of rehabilitation. Philadelphia, Lippincott Williams \&Wilkins, pp 153-181.

24. Hospital records of Sohag University, (20132014).

25. Irimia, O., (2012): Physical Therapy Clinical Handbook for PTAs, Part 5 cardiopulmonary interventions, pp. 308-315.

26. Khalil, Y., Mustafa, E., Youssef, A., Imam, M., \& El Behiry, A., (2012): Neuromuscular dysfunction associated with delayed weaning from mechanical ventilation in patients with respiratory failure. Alexandria Journal of Medicine, Vol 48, pp. 223-232.

27. Mączka, A., Kowalski, I., \& Onichimowski, D., (2011): Pulmonary rehabilitation within intensive care units exemplified by traffic collisions casualties. Polish Annals of Medicine, Vol 18(1), pp. 66-75.

28. Mackenzie, C., Shin, B., Hadi, F., Imle, P., (2011): Changes in total lung-thorax compliance following chest physiotherapy. Anaesthesia Analgesia, vol 59, pp. 207-210.

29. Make, B., Gilmartin, M., Brody, J., Snider, G., (1984): Rehabilitation of Ventilator-Dependent Subjects with Lung Diseases. The Concept and Initial Experience. Chest, Sep;86(3):358-65. Downloaded From: http://journal.publications.chestnet.org/ on $12 / 28 / 2014$

30. Malkoc, M., Karadibak, D., \& Yıldırım, Y., (2009): The effect of physiotherapy on ventilatory dependency and the length of stay in an intensive care unit. International Journal of Rehabilitation Research, Vol 32(1), pp. 85-88.

31. Maskara, S., \& Sen, N., (2000): Correlation between lung injury score and serum albumin levels in patients at risk for developing acute lung injury. Nutrition, Vol 16 (1), pp. 91-94.

32. Mendez-Tellez, A., Nusr, R., Feldman, D., Needham, D., (2012): Early Physical Rehabilitation in the ICU: A Review for the Neurohospitalist. Neurohospitalist, vol 2(3), pp.96-105.

33. Moreira, F., Teixeira, C., Savi, A., \& Xavier, R., (2015): Changes in respiratory mechanics during respiratory physiotherapy in mechanically ventilated patients. Rev. Bras. Ter. Intensive, vol 27 (2), pp.155-160.

34. Morris, P., Goad, A., Thompson, C., Taylor, K., Harry, B., Passmore, L., Ross, A., Baker, S., Sanchez, M., Penley, L., Howard, A., Dixon, L., Leach, S., Small, R., Hite, R., Haponik, E., (2008): Early intensive care unit mobility therapy in the treatment of acute respiratory failure. Crit Care Med, vol 36, pp. 2238-2243.

35. Müller, M., Strobl, R., \& Grill, E., (2011): Goals of patients with rehabilitation needs in acute hospitals: goal achievement is an indicator for improved functioning, J Rehabil Med, vol 43, pp.145-150.

36. Needham, D., Korupolu, R., Zanni, J., Pradhan, P., Colantuoni, E., Palmer, J., Brower, R., \& Fan, E., (2010): Early physical medicine and rehabilitation for patients with acute respiratory failure: a quality improvement project. Arch Phys Med Rehabil, vol 91, pp. 536-542.

37. Paratz, J., Lipman, J., \& McAuliffe, M., (2002) Effect of manual hyperinflation on hemodynamics, gas exchange, and respiratory mechanics in ventilated patients. J Intensive Care Med. Vol 17 (6), pp.317-324.

38. Parker, A., \& Needham, M., (2013): the Importance of Early Rehabilitation and Mobility in the ICU. Society of critical care medicine. Available at: http://www.sccm.org/Communications/CriticalConnections/Archives/Pages/Importance-EarlyRehabilitation-Mobility-ICU.aspx. Accessed at 26 Dec. 2016.

39. Perry, A., Potter, A., Ostendorf, W., (2015) Nursing Interventions \& Clinical Skills, $6^{\text {th }}$ edition, Chapter 14 promoting oxygenation, pp. 356-441

40. Rooney, A., (2013): Improving recovery with critical care rehabilitation. Nursing Times. vol 109 26, pp. 23-25.

41. Routsi, C., Gerovasili, V., Vasileiadis, I., Karatzanos, E., Pitsolis, T., Tripodaki, E., Markaki, V., Zervakis, D., \& Nanas, S. (2010): Electrical muscle stimulation prevents critical illness polyneuromyopathy: a randomized parallel intervention trial with consumer summary. Crit Care.14: R74.

42. Schweickert, W., Pohlman, M., Pohlman, A., Nigos, C., Pawlik, A., Esbrook, C., Spears, L., 
Miller, M., Franczyk, M., Deprizio, D., Schmidt, G., Bowman, A., Barr, R., McCallister, K., Hall, J., \& Kress, J., (2009): Early physical and occupational therapy in mechanically ventilated, critically ill patients: a randomized controlled trial. Lancet. vol 373, pp. 1874-1882.

43. Sessler, C., Gosnell, M., \& Grap, M., (2002) The Richmond Agitation-Sedation Scale: validity and reliability in adult intensive care patients. Am J Respir Crit Care Med, vol 166, pp. 1338-1344.

44. Sharma, B., \& Singh, V., (2011): Pulmonary rehabilitation: An overview. Official publication of Indian Chest Society Lung India. Vol 28(4), pp. 276-284.

45. Stiller, K., Phillips, A., \& Lambert, P., (2004): The safety of mobilisation and its effects on haemodynamic and respiratory status of intensive care patients. Physio Theory Pract, vol 20, pp.175-185.

46. The Team Study Investigators, (2015): Early mobilization and recovery in mechanically ventilated patients in the ICU: a bi-national, multi-centre, prospective cohort study. Critical Care. 19:81.

47. Vitacca, M., Paneroni, M., Peroni, R., Barbano, L., Dodaj, V., Piaggi, G., Vanoglio, F., Luisa, A., Giordano, A., \& Ceriana, P., (2014): Effects of a Multidisciplinary Care Program on Disability, Autonomy, and Nursing Needs in Subjects Recovering From Acute Respiratory Failure in a Chronic Ventilator Facility. Respiratory Care. vol 59(12), pp. 18631871.

48. Vollman, K., (2013): Understanding critically ill patient's hemodynamic response to mobilization: Using the evidence to make it safe and feasible.
Critical Care Nursing Quarterly, Vol 36(1), pp.1727.

49. Ware, L., (2010): Pathophysiology of acute lung injury and the acute respiratory distress syndrome. Respir Crit Care Med. vol 27(4), pp.337-349. 